\title{
Application of a New Processing Method to Post-LDL-apheresis Data
}

\author{
FRANCO CORSINI ${ }^{\mathrm{a}}$, ARRIGO F. G. CICERO ${ }^{\mathrm{b}, *}$, ANTONIA GIANNUZZI $^{\mathrm{c}}$ and ANTONIO GADDI ${ }^{\mathrm{b}}$ \\ ${ }^{\mathrm{a}}$ Medical and Surgical Paediatric Sciences Complex Unit, University of Bologna, Italy; ${ }^{\mathrm{b}}$ Clinical Medicine and Applied Biotechnology Department \\ “D. Campanacci”, Atherosclerosis centre "G. Descovich”, University of Bologna, Orsola-Malpighi Hospital, Via Massarenti 9, 40138 Bologna, Italy; \\ ${ }^{\mathrm{c} C l i n i c a l}$ Medicine and Applied Biotechnology Department "D. Campanacci”, University of Bologna, Italy
}

(Received 18 October 2001; In final form 29 July 2002)

\begin{abstract}
Our aim was to elaborate a method to optimise treatment intervals for the individual low-density lipoprotein (LDL)-apheresis treated patients. After each treatment, plasma LDL concentrations show a time-related increase with a decreasing speed until a maximum level. We searched to interpret the postLDL-apheresis experimental data trend as the physical process that produces the observed curve, so that the fitting presupposed theoretical function is a direct consequence of the physic process, because to establish the better time. Applying the proposed fitting method to a succession of 15 samples obtained from the mean of six plasmapheresis executed on five different subjects, small estimate standard error $(5 \mathrm{mg} / \mathrm{dl})$ and relative error $(1.7 \%)$ with a dispersion evidently related to the experimental error were observed. Obviously, applying the same method to a single case, the dispersion is more marked (relative error $<5 \%$ ), with a SE of $10-13 \mathrm{mg} / \mathrm{dl}$, even though the aspect of a casual phenomenon is conserved. Our physical interpretation appears to be a practical model to predict the LDL-rebound kinetic of the single patient.
\end{abstract}

Keywords: Fitting method; LDL-apheresis; LDL kinetic; Mathematical model

\section{INTRODUCTION}

Low-density lipoprotein (LDL)-apheresis is an extracorporeal procedure for selectively removing apolipoprotein B containing lipoproteins from the blood. Its utility is well recognised in those patients in whom the maximal pharmacological treatment (high doses of Hydroxy-Methyl-Glutaryl-CoA reductase inhibitors associated with fibrates or ionic exchange resins) is not able to obtain a sufficient lipid lowering. In the United States of America, the Food and Drug Administration has established clear guidelines for the LDL-apheresis employing: homozygous familial hypercholesterolemia (FH) patients with LDL-Cholesterol level $>500 \mathrm{mg} / \mathrm{dl}$, patients with coronary artery disease and LDL-C level $>200 \mathrm{mg} / \mathrm{dl}$ and all other subjects with LDL-C level $>300 \mathrm{mg} / \mathrm{dl}$ (Gordon and Saal, 1997). There are three principal LDL-apheresis techniques: immunoadsorption with polyclonal apolipoprotein B antibodies (Stoffel et al., 1981; Richter et al., 1993), heparin induced extracorporeal LDL precipitation (Eisenhauer et al., 1987; Seidel et al., 1991) and dextrane sulfate adsorption (Homma et al., 1986; Mabuchi et al., 1987). All the techniques have demonstrated their safety and long-term efficacy on coronary and extracoronary artery disease (Richter et al., 1996; Thiery Seidel, 1998) treatment. Besides the LDL concentration decreasing action, the same technique is even able to reduce the plasma level of other cardiovascular risk factors such as lipoprotein $(a)$ (Armstrong et al., 1989; Gruber et al., 1992) and fibrinogen (Jaeger et al., 1997). Therefore, after an apheresis treatment, the plasma concentrations of different substances show a time-related increase with a decreasing speed until a maximum level. The aim of our research is to interpret the post-apheresis experimental data on the basis of the physical process that produces the observed curve so that the theoretical function presupposed to the fitting does not rely on an arbitrary choice, but it is a direct consequence of the same physical process itself. The validity of the presupposed

*Corresponding author. Tel.: + 39-516364920. Fax: + 39-59762089. E-mail: afgcicero@tiscalinet.it 
basic physical hypothesis as well as the goodness of the fitting procedure will be judged according to the obtained results.

\section{METHOD}

The described model is the result of the concomitant entrance of a substance (S) in the blood stream and its disappearance through catabolic pathways. To estimate the $\mathrm{S}$ quantity that enters/leaves the plasma per volume unit in the time unit, we make two hypotheses to be verified on the basis of experimental results: (1) in each time unit the S quantity that enters the system is constant, independent from the time elapsed from the last apheresis (this quantity remains constant even after $\mathrm{S}$ has reached its maximum level); (2) in the time unit $\mathrm{S}$ disappearing quantity is proportional to its plasmatic concentration on the basis of a constant. From these two hypotheses, we can write down the equation $y=(P-k y) \Delta t$ in which $y$ represents the $\mathrm{S}$ quantity per blood volume unit $(\mathrm{mg} / \mathrm{dl})$, $\Delta y$, the variation of $\mathrm{S}$ quantity in the time unit, $P$ is the $\mathrm{S}$ quantity that enters the system in the time unit, $k$ is the proportionality coefficient between the $\mathrm{S}$ disappearing quantity and the $\mathrm{S}$ actual quantity. If the $\Delta t$ time interval tends to zero, and if we integrate this equation, we obtain the equation $y(t)=P / k\left(1-\mathrm{e}^{-k t}\right)$ in which $y$ is timedependent.

In reality, apheresis is not able to eliminate all $\mathrm{S}$ from plasma, so even immediately after apheresis $\mathrm{S}$ concentration is never zero and the experimental times do not coincide with those of the curve natural temporal co-ordinate. To reckon virtual $t_{0}$ when $\mathrm{S}(t)$ is zero, it is necessary to move backwards with subsequent extrapolations beginning from the real concentrations observed from the first sample after apheresis. Mathematically, the time co-ordinate that has the zero point at the apheresis end is fictitious, while the time co-ordinate in which for $t=0, y=0$ is the natural time co-ordinate of the function. Besides, the distance of experimental $t_{0}$ where $y \neq 0$ and $t_{0}=0$ with $y=0$ could be considered as an efficiency index of the plasmapheresis procedure. Thus, the numerical values to be introduced in the fitting of the experimental data are the times $t(j)$ measured in the natural co-ordinate of the theoretical curve and the values of the parameters $k$ and $P$. Since the analytical determination of these values is not possible, it is necessary to apply an iterative nonlinear fitting method. We assign to the fundamental parameters $t$ and $k$ numeric values growing with an initially assigned step which is then varied according program in an a priori defined way. A similar procedure is carried out for $k$ : after definition of a minimum value, $k$ is increased by programmed steps to find the best approximation of data for the given $t(j)$. The asymptotic value $A=P / k$ of the curve is directly calculated through the least squares method applied on equation $y(t)=P / k\left(1-\mathrm{e}^{-k t}\right)$ from the given values of $t$ and $k$.
This is possible because the equation is linear with respect to $P$. Finally, the $y c(j)$ are calculated and compared with the experimental $y(j)$. The criterion to evaluate the approximation quality is the resulting standard error of estimate (erm) between experimental and calculated values of concentration $\left[\mathrm{erm}=\sqrt{\sum[y s(j)-y c(j)]^{2} /(n-2)}\right]$.

The flow-chart shown in Figure 1 explains, more precisely, the whole procedure.

On the basis of the final calculated values, it is also possible to find out the time $\left(t_{\mathrm{au}}\right)$ of the first postapheresis sample corrected for the natural co-ordinate, and the time and concentration $\left(t_{\mathrm{pa}}\right.$ and $\left.y t_{\mathrm{pa}}\right)$ at the plasmapheresis end. It is important to observe that $t_{\mathrm{au}}$ $[y c(2)$ on the curve] is in general not equal to the experimental value related to the same time. In fact, the literature often excludes the first experimental datum from the fitting, so that the experimental error of a single value is transferred on the whole theoretical function.

Particularly interesting, from a clinical point of view, is the mean substance concentration $\left(y_{\mathrm{m}}\right)$ in the postapheresis observed range of time. In fact, this procedure is used to maintain, under lower values, the plasmatic concentration of the substance able to cause damage. This mean is calculated through the integration of the values of the substance between the end of plasmapheresis $\left[t_{\mathrm{pa}}\right]$ and the final observation time $[t(n)]$, divided by the total considered time $\left[t(n)-t_{\mathrm{pa}}\right]$ :

$$
\begin{aligned}
y_{\mathrm{m}} & =\left[\int_{t_{\mathrm{pa}}}^{t(n)} A\left(1-\mathrm{e}^{-k \mathrm{~S}}\right) \mathrm{d} s\right] /\left[t(n)-t_{\mathrm{pa}}\right] \\
& =A\left[1-\left(\mathrm{e}^{-k t_{\mathrm{pa}}}-\mathrm{e}^{-k t(n)}\right) /\left\{k\left[t(n)-t_{\mathrm{pa}}\right]\right\}\right]
\end{aligned}
$$

\section{RESULTS}

A test of efficiency of the fitting procedure in reproducing artificial functions submitted to initial truncation similar to the experimentally observed ones (or even purposely magnified in comparison with these) gave very good results, as shown in Figure 2. Here, the original (simulating the experimental) data and the calculated ones, owing to the non-existence of experimental errors, were nearly equal so that their graphical images are practically superimposed. In the application to experimental data, a good concordance between fitting results and observed data was evidenced. Applying the proposed fitting method to a succession of 15 samples obtained from the mean of six plasma dextrane LDL apheresis executed on five different subjects, small estimate standard error $(5 \mathrm{mg} / \mathrm{dl})$ and relative error (absolute mean error/mean $\mathrm{S}$ concentration $=1.7 \%$ ) with a dispersion that appears to be evidently related to the experimental error were observed (Figure 3). 


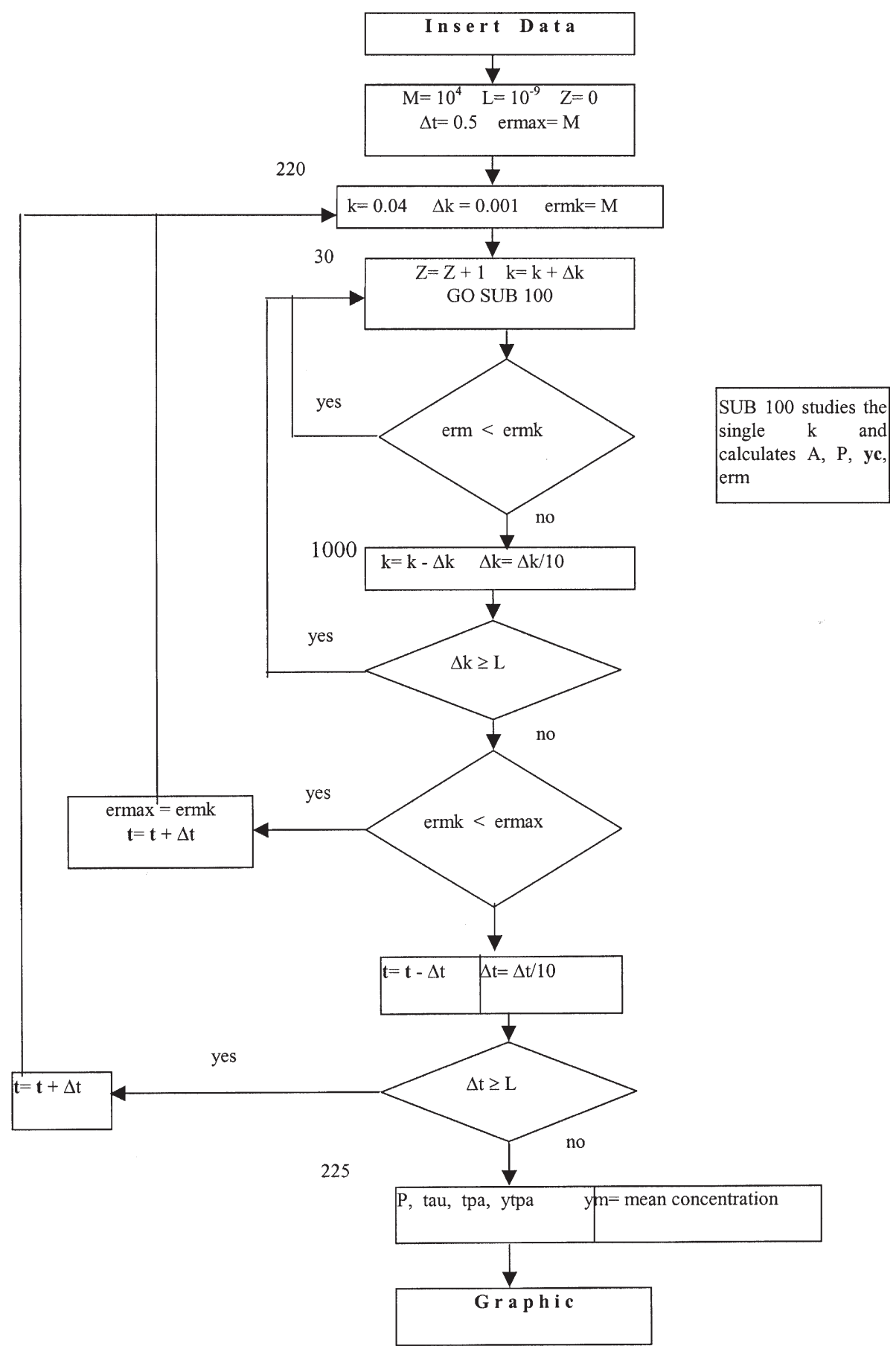

FIGURE 1 Flow chart explaining the procedure to find the theoretical values on the basis of the experimental data (in bold the vectorial terms).

Obviously, applying the same method to a single case, the dispersion is in general more marked (relative error until 5\%), with a standard error varying from 10 to $13 \mathrm{mg} / \mathrm{dl}$, even though the aspect of a casual phenomenon is conserved.

The time to analyse a curve that contains 15 experimental data is of a few seconds using a Quick Basic software on an old Epson portable computer (486 microprocessor, $50 \mathrm{MHz}$ ).

\section{DISCUSSION}

Despite the numerous reports describing the efficacy of LDL-apheresis in the treatment of hypercholesterolemic patients, opinion diverge considerably on the proper timing of treatments, a bi-weekly interval between two successive procedures being most commonly selected (Gordon and Saal, 1997). In fact, it is still not clear what is the relative benefit of shorter versus longer intervals 


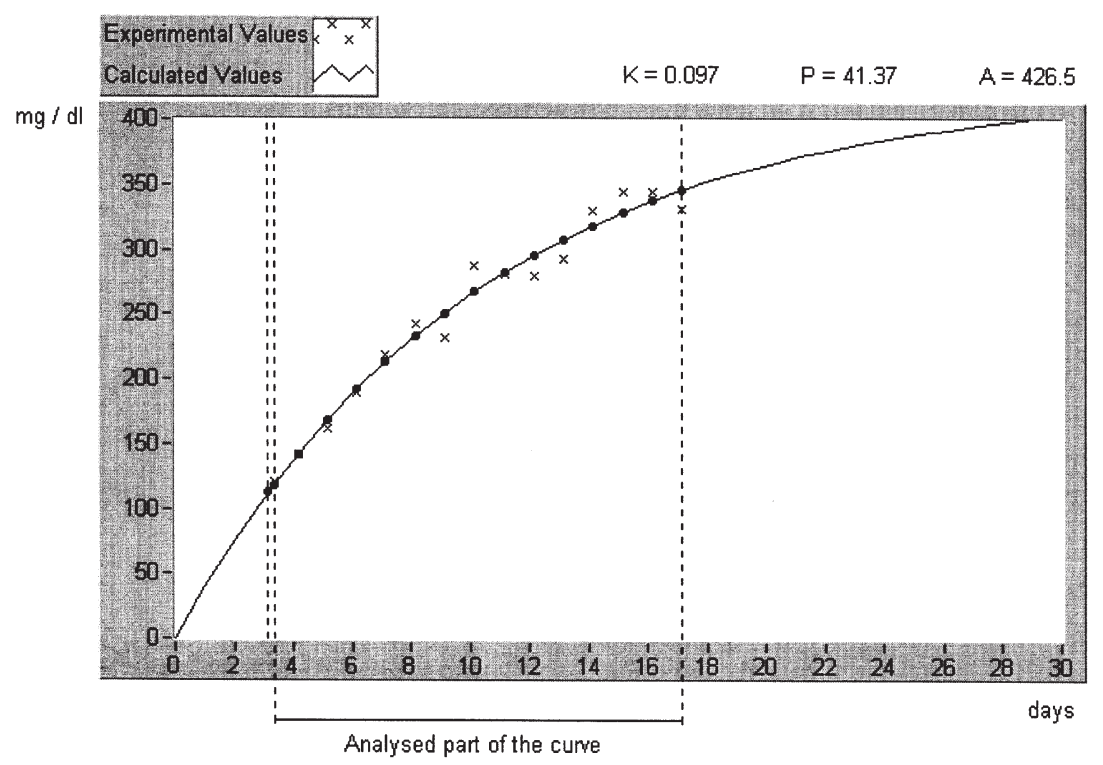

FIGURE 2 Test of the efficiency of the fitting method in reproducing initially truncated artificial functions.

between treatments (Kroon et al., 2000). After an apheresis treatment, plasmatic concentrations of various substances show a time-related increase with a decreasing speed until a maximum level: this behaviour is very similar to other processes happening on different physical and physiological basis. Therefore, the experimental data obtained after the post-LDL-apheresis have been differently read. After bi-logarithmic transformation, Franceschini et al. (1991) found out that the evaluation of the so determined parameters is useful to estimate the individual LDL-concentration trend related to the different times of the treatment execution. Kochinke et al. (1988) observed the likeness of the curve describing the LDL rebound and the curve of the enzymatic reaction versus the substrate concentration and apply the Michaelis-Menten formula after changing the symbols. However, these different ways of reading the experimental data have a merely formal nature, because they do not describe the phenomenon of the rebound starting from the process that produces its trend-though in their dynamics comprehensible according to the Langer et al. (1972) proposals and the Kochinke et al.'s confirmations. Another two-compartment cholesterol kinetic model has been proposed by Yashiro et al. (1994). Instead, our aim is to interpret the experimental data on the basis of the physical process that produces the observed curve so that the presupposed theoretical function is a direct consequence of the physical process itself. It is evident

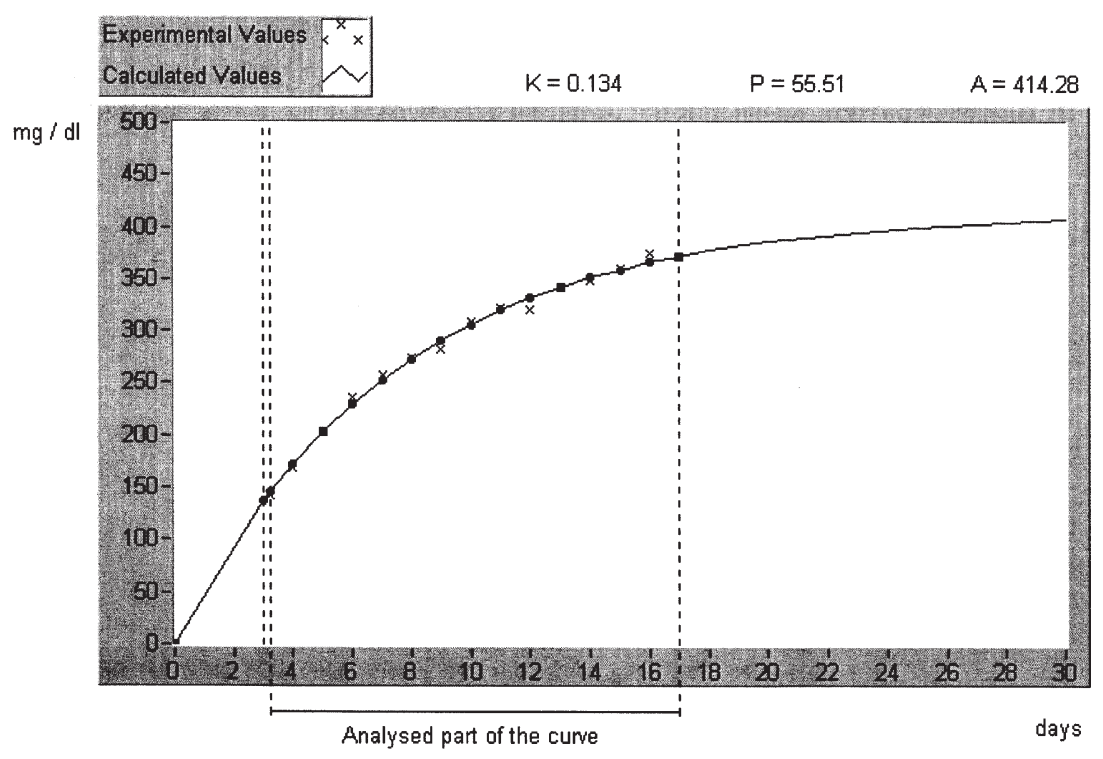

FIGURE 3 Application of the proposed fitting method to a succession of 15 samples obtained from the mean of six plasmapheresis executed on five different subjects. 


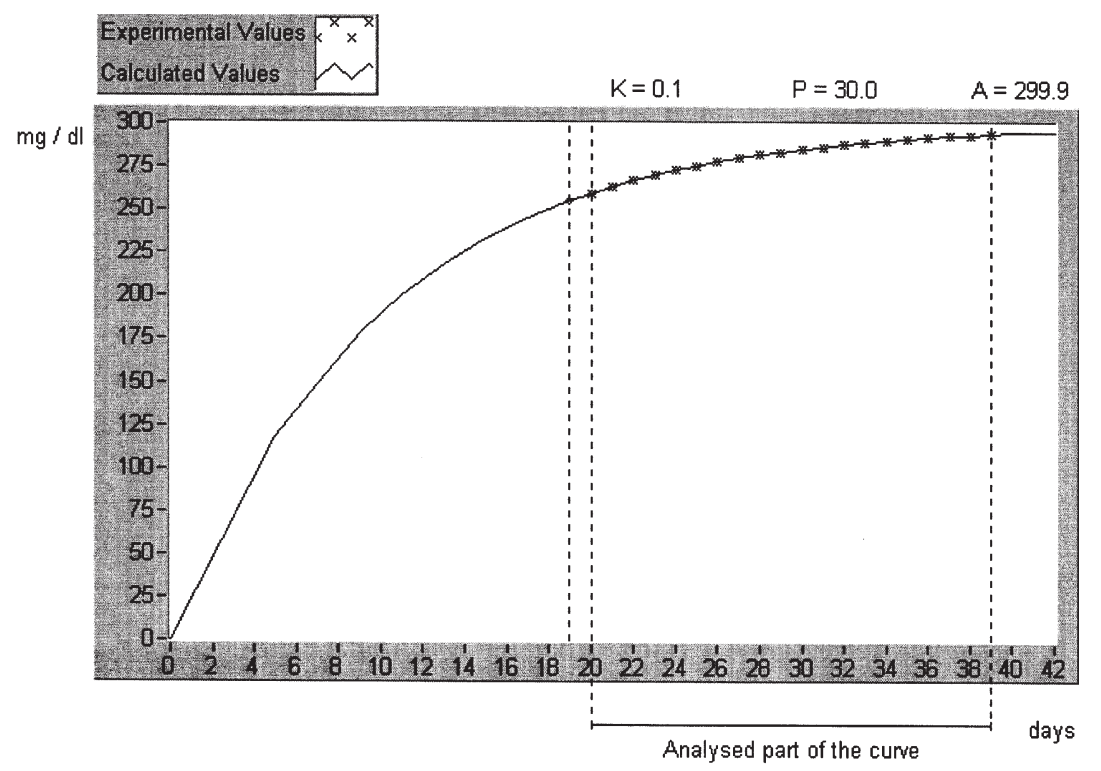

FIGURE 4 Application of the proposed fitting method to a single patient treated with LDL-apheresis.

that a theoretical function that approximately calculates the mean substance concentration in the post-apheresis time can be useful to make predictions and statistical considerations of clinical significance. This is especially true for patients in primary prevention who have no symptoms and may question the necessity of too frequent extra-corporeal treatments. Moreover, optimising treatment intervals for the individual patient will lead to savings both of direct and indirect costs.

The metabolic processes that modify the plasma lipoprotein concentration are really complex (Beltz et al., 1985). Nevertheless, the answer obtained from the experimental data fitting during the return to an equilibrium condition after plasmapheresis let us suppose that the basic physical process is regulated from a linear dynamic as presupposed for the tested fitting method (Figure 4). More fine regulation effects cannot be excluded, but their individuation could be difficult owing to the not eliminable physiological and methodological casual variability that easily could mask these effects.

In conclusion, biological models of the post-apheresis LDL-rebound kinetics are not easily applicable to the clinical practice, because the necessity of a more complete analysis of several compartments, not excluding the use of invasive investigation means. Our physical-mathematical interpretation of the experimentally obtainable data appears to be a practical model to understand the LDLrebound kinetic of the single patient without using radioactive markers. The same method could be applied to other lipoprotein fractions as $\mathrm{Lp}_{(\mathrm{a})}$ and to fibrinogen as well as to all the substances removed from plasma trough an apheretic treatment.

\section{References}

Armstrong, V.W., Schleff, J., Thiery, J., Muche, R., Schuff-Werner, P., Eisenhauer, T. and Seidel, D. (1989) "Effect of HELP-LDL-apheresis on serum concentration of human lipoprotein(a): kinetic analysis of the post-treatment return to baseline levels", European Journal of Clinical Investigation 19, 235-240.

Beltz, F., Kesaniemi, Y.A., Howard, B.V. and Grundy, S.M. (1985) "Development of an integrated model for analysis of the kinetics of apolipoprotein B in plasma very low density lipoproteins, intermediate density lipoproteins and low density lipoproteins", Journal of Clinical Investigation 76, 575-581.

Eisenhauer, T., Armstrong, V.W., Wieland, H., Fuchs, C., Scheler, F. and Seidel, D. (1987) "Selective removal of low density lipoprotein (LDL) by precipitation at low $\mathrm{pH}$ : first clinical application of the HELP system", Klinical Wochenschrift 65, 161-165.

Franceschini, G., Busnach, G., Calabresi, L., Chiesa, G., Gianfranceschi, G., Zoppi, F., Minetti, L. and Sirtori, C.R. (1991) "Predictability of low density lipoprotein levels during apheretic treatment of hytpercholesterolemia", Europran Journal of Clinical Investigation 21, 209-214.

Gordon, B.R. and Saal, D.S. (1997) "Clinical experience and future directions for low density lipoprotein apheresis in the United States", Therapeutical Apheresis 1(3), 249-252.

Gruber, C., Swoboda, K., Pidlich, J., Gottsaumer-Wolf, M., SunderPlassmann, G., Pamberger, P., Jansen, M., Derfler, K. and Widhalm, K. (1992) "Reduction of Lipoprotein(a) by immunospecific LDL apheresis", In: Gotto, Jr, A.M., Mancini, M., Richter, W.O. and Schwandt, P., eds, Treatment of Severe Dyslipoproteinemia in the Prevention of Coronary Heart Disease 4th International Symposium, Munich, 1992, pp 219-222.

Homma, Y., Mikami, Y., Tamachi, H., Nakaya, N., Nakamura, H., Araki, G. and Goto, Y. (1986) "Comparison of selectivity of LDL removal by double filtration an dextran-sulfate cellulose column plasmapheresis", Atherosclerosis 60, 23-28.

Jaeger, B.R., Meiser, B., Nagel, D., Ueberfuhr, P., Thiery, J., Brandle, U., et al. (1997) "Aggressive lowering of fibrinogen and cholesterol in the prevention of graft vessel disease after heart transplantation", Circulation 96(2), 154-158.

Kochinke, F., Von Baeyer, H., Schwaner, I., Graefenhahn, H., Nold, A., Schwerdtfeger, R. and Schwartzkopf, W. (1988) "Modelling of LDLapheresis: system efficiency and rebound kinetics", Plasma Therapeutic and Transfusional Technology 9, 35-44.

Kroon, A.A., van't Hof, M.A., Demacker, P.N.M. and Salenhof, A.F.H. (2000) "The rebound of lipoproteins fter LFL-apheresis. Kinetics and estimation of mean lipoprotein levels", Atherosclerosis 152, $519-526$.

Langer, T., Strober, W. and Levy, R.I. (1972) "The metabolism of low density lipoproteins in familial type II Hyperlipoproteinemia", Journal of Clinical Investigation 51, 1528-1532.

Mabuchi, H., Michishita, I., Takeda, M., Fujita, H., Koizumi, J., Takeda, R., et al., (1987) "A new low density lipoprotein apheresis system using two dextran-sulfate cellulose columns in an automated column 
regenerating unit (LDL-continuous apheresis)", Atherosclerosis $\mathbf{6 8}$, 19-25.

Richter, W.O., Jacob, B.G., Ritter, M.M., Suhler, K., Vierneisel, K. and Shwandt, P. (1993) "Three-year treatment of familial heterozygous hypercholesterolemia by extracorporeal LDL-immunoadsorbtion with polyclonal apolipoprotein B antibodies", Metabolism 42, $888-894$.

Richter, W.O., Donner, M.G. and Schwandt, P. (1996) "Long-term effect of LDL-apheresis on coronary heart disease", Acta Angiologica 2(2), $147-151$.

For the HELP Study GroupSeidel, D., Armstrong, V.W. and SchuffWerner, P. (1991) "The HELP-LDL-apheresis Multicenter Study, an angiographically assessed trial on the role of LDL-apheresis in the secondary prevention of coronary heart disease. I. Evaluation of safety and cholesterol lowering effects during the first 12 month", European Journal of Clinical Investigation 21, $375-381$.

Stoffel, W., Greve, G.V. and Borberg, H. (1981) "Application of specific extracorporeal removal of low density lipoprotein in familial hypercholesterolemia", Lancet 2, 1005-1011.

Thiery, J. and Seidel, D. (1998) "Safety and effectiveness of long term apheresis in patient at high risk", Current Opinion in Lipidology 9 , $521-526$.

Yashiro, M., Muso, E., Matsushima, M., Nagura, R., Sawanishi, K. and Sasayama, S. (1994) "Two-compartment model of cholesterol kinetiks for establishment of treatment strategy of LDL-apheresis in nephrotic hypercholesterolemia", Blood Purification 12(6), $317-326$. 


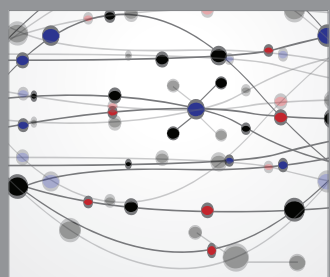

The Scientific World Journal
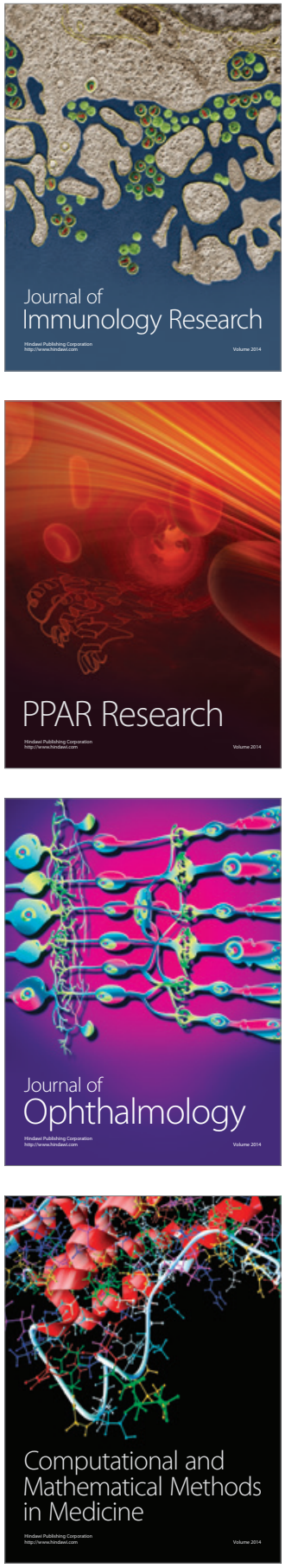

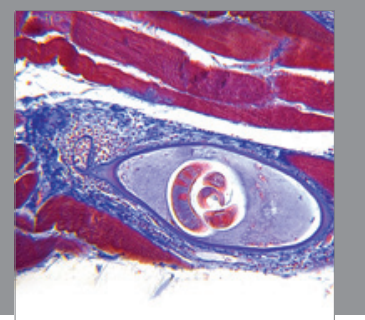

Gastroenterology

Research and Practice
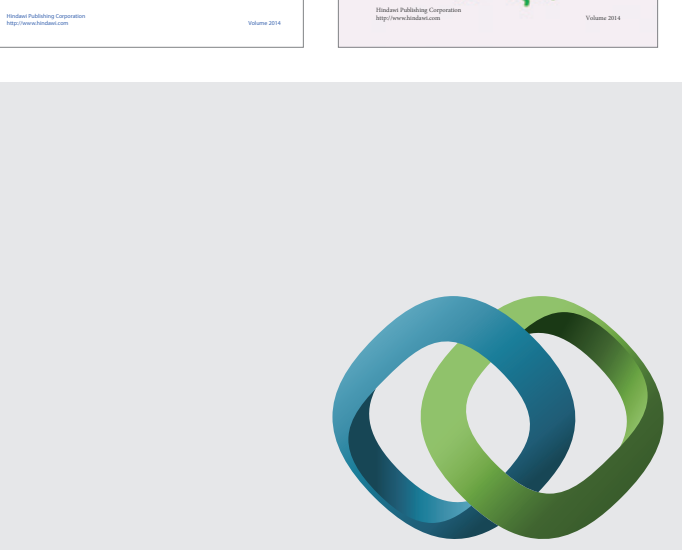

\section{Hindawi}

Submit your manuscripts at

http://www.hindawi.com
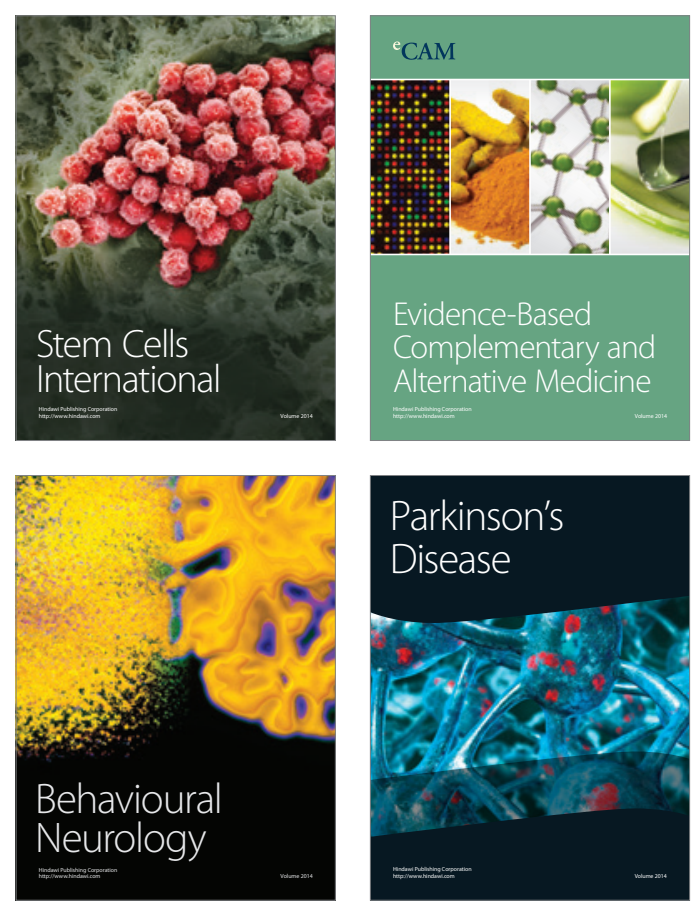

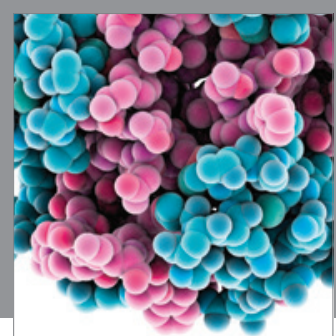

Journal of
Diabetes Research

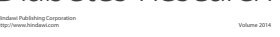

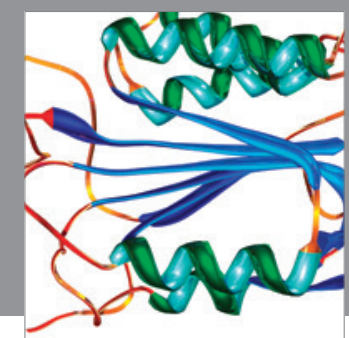

Disease Markers
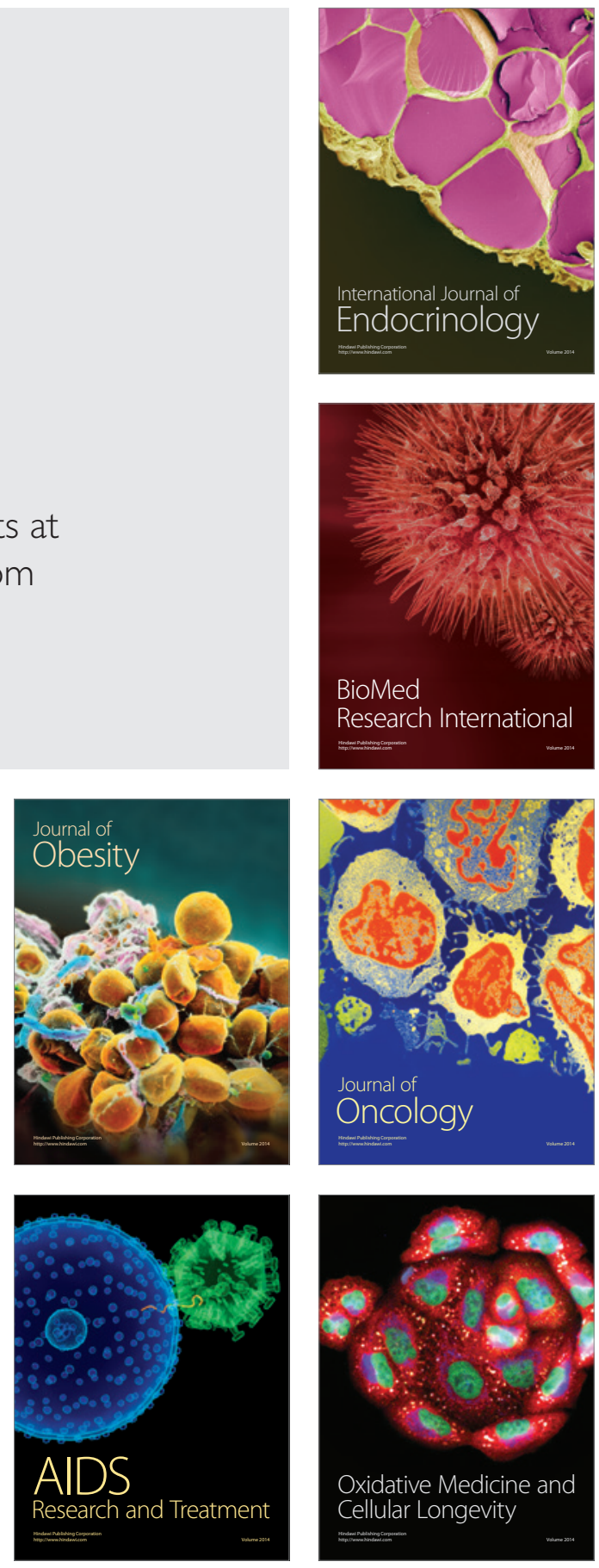